\title{
Power Available from a Depth-averaged Simulation of a Tidal Turbine Array
}

\author{
C. R. Vogel ${ }^{\mathrm{a}, *}$, R. H. J. Willden ${ }^{\mathrm{a}}$, G. T. Houlsby ${ }^{\mathrm{a}}$ \\ ${ }^{a}$ Department of Engineering Science, University of Oxford, OX1 3PJ, United Kingdom
}

\begin{abstract}
The power available to a tidal stream turbine fence depends on the turbine resistance, the mass flux through the array, as well as the flow speed variation within the array. Depth-averaged simulations are often used to analyse the power available at a site, where the array is modelled as a region of enhanced flow resistance. Without special treatment this method can lead to errors when estimating the power available to the array as the simulated velocity at the turbine plane, and therefore the inferred power, is a spatially averaged value and does not capture flow speed variation within the array; true power being dependent on through-turbine velocity rather than array area-averaged velocity. Linear momentum theory is used to represent the turbines, which by equating the thrust of the numerically simulated array and the analytically modelled turbines enables the true power available to the array to be correctly evaluated. This power is shown to always be less than the erroneous power inferred from the simulated array area-averaged flow velocity. Array end effects, due to the skewed flow passing through the simulated turbine array region, are shown to be particularly important when estimating the power available to short and high-thrust arrays.
\end{abstract}

Keywords: Tidal power, Tidal currents, Tidal turbine arrays, Depth-averaged simulations

1 The global tidal stream resource has attracted attention in recent years as a potential source of predictable renewable energy, and a large number of turbines deployed in an array or farm configuration will be required to generate a significant amount of power. Recent analytic models and numerical simulations of arrays partially spanning wide channels have shown that there are two scales of flow to be considered for turbine fences: the device-scale flow, the flow around an individual turbine and its wake and remixing with the device scale bypass; and the array-scale flow, the flow around the array and its wake and remixing with the bulk flow through the channel $[9,10]$. Flow diversion around the array reduces the mass flux through the array which then provides the upstream boundary condition to the device-scale flow problem.

Power is removed from the flow in several stages, and a distinction is made between the power available to a device for conversion into mechanical energy, which is the inviscid limit of the shaft power of a turbine [1], and the power dissipated in mixing processes in the wake. The total power removed from the flow is the sum of the available power and the power dissipated in the wake. Power definitions may be further separated into device and array scales, where the device power is the power available to a single device, and is a function of device-scale thrust and flow speed. The array power comprises the total power available to the tidal devices as well as the power dissipated through device-scale wake mixing. The array power is therefore necessarily greater than the power available to the devices in the array. Estimating the power available to an array thus requires both the device- and array-scale flows to be resolved. The total power removed from the flow is then the sum of the array power and the power lost in array-scale mixing with the channel flow.

The grid resolution in three-dimensional simulations, used to study small groups of tidal devices, ensures that both the devices and their wakes are resolved, allowing the available power to be estimated (see [8]).

${ }_{21}$ However, the computational expense of simulating many tidal devices means that two-dimensional simulations

${ }^{*}$ Corresponding author

Email address: christopher.vogel@eng.ox.ac.uk (C. R. Vogel) 
are preferred [2]. The flow is typically described in terms of depth-averaged quantities in these simulations, and representing device-scale flow can be challenging as the grid cells may be larger than the size of a device and/or array [11], making it difficult to determine what local and array flow speeds may be reliably used as reference values.

Relating the power removed from the depth-averaged simulation to the available device power, the maximum power that can be extracted at the turbine plane, is challenging as the relationship between the available power and the total power removed from the flow is not straightforward [3]. If turbine design data are available, the relationship can be determined through experiments, numerical simulation, and/or analytic models. Alternatively, an upper bound on the available array power can be established using idealised actuator disks, which act as perfectly efficient momentum extractors; examples include [4], [9] and [11]. The relationship between the array flow speed and disk thrust and power may be determined analytically or through numerical simulations.

This paper develops analytic sub-models for depth-averaged simulations based on linear momentum actuator disk theory (LMADT), which considers momentum and mass conservation in the core and bypass flows past actuator disks, to determine the maximum power available to an actuator disk array. We begin by reviewing challenges in depth-averaged modelling device- and array-scale flows and the different characteristics of the two flows. Analytic corrections are developed based on rigid-lid ([5]) and open channel ([3] and [13]) actuator disk analyses. Drag arising from the support structure is neglected, although this can be modelled as an additional resistance to the flow.

\section{Challenges in Depth-averaged Modelling of Tidal Turbine Arrays}

Detailed three-dimensional models are required to resolve the complexities of the flow around a tidal turbine and in its wake. Inevitably, some of these features are lost when models of reduced dimension are used, such as depth-averaged simulations. Thus, the present challenge is how to appropriately account for the unresolved components of the flow and their effect on the thrust and power of the turbine. This paper address three questions arising from this challenge:

1. What is the minimum scale in the flow that can be accurately represented?

2. What is an appropriate reference velocity for parameterising turbine performance?

3. How can momentum be removed from the depth-averaged flow in a manner that is consistent with the underlying three-dimensional case being modelled?

The first question introduces the challenges of developing a numerically stable, grid-resolution independent representation of the turbines, as well as identifying flow phenomenon which cannot be adequately represented in a reduced-order model. The second challenge is to identify an appropriate depth-averaged reference velocity in the numerical simulation, recognising that the flow speed through the turbines may be different to the depth-averaged flow speed at the turbine location, as well as numerical issues with referencing velocities elsewhere in the domain due to parallelisation of the simulation. The third question is closely related to the first two, in which it is necessary to ensure that the simulation is, as much as possible, the depth-averaged equivalent of the three-dimensional process being modelled, by removing the same amount of momentum from the flow. Each challenge is briefly discussed below.

Numerical stability in the turbine simulation region is very important, as erroneous velocities due to numerical instabilities, such as those arising from the magnitude of applied forcing terms and the discretisation method, can result in significant errors in the prediction of turbine thrust and power. As discussed in Section 4 , the Discontinuous Galerkin Finite Element Method (DG-FEM) allows step discontinuities in the fluid depth and velocity between adjacent cells, allowing the effects of momentum extraction by the turbines to be determined. Spurious oscillations may develop in the presence of a step discontinuity in a Continuous Galerkin Finite Element (CG-FEM) implementation, requiring a slightly different modelling approach. The numerical representation of the turbines should be grid-independent, insofar as the predicted thrust and power are largely independent of grid size on a converged grid, such as the approach described in [7]. 
One challenge in depth-averaged modelling is that the bypass flow around the turbine is not resolved in the vertical dimension, leading to higher depth-averaged velocities at the turbine plane than are seen through the turbine itself, leading to an over-prediction of the thrust and power. It is also challenging to identify an appropriate reference velocity in the domain upstream of the turbines, as the flow speed will be affected by the resistance the turbines apply to the flow, and variations in the domain bathymetry and the influence of boundary conditions makes it difficult to determine an appropriate velocity reference location. Using a non-local velocity may not be computationally desirable if values must be passed between sub-domains in a parallelised model [7]. It is therefore important also to determine a robust and numerically stable method for defining reference flow speeds to determine the turbine performance.

Finally, it is important to correctly represent the three-dimensional turbines in the depth-averaged simulation. The momentum removed from the depth-averaged flow should correspond to the thrust applied to the flow by the array of turbines, a challenge which is related to that of defining an appropriate reference velocity. This requires either a modified bottom drag coefficient (e.g. [7]), or the application of a depth-averaged force as described below.

Furthermore, it is also important to conserve the blockage ratio, the ratio of turbine frontal area to flow passage cross-sectional area, of the three-dimensional array of turbines in the depth-averaged simulation. Garrett and Cummins [5] showed that the power available to a turbine was, in part, a function of the blockage ratio of the turbine. Similarly, the power available to an array is a function of the blockage ratio of the array in the larger channel. In the case of an axial flow turbine of diameter $d$, which is necessarily less than the depth of the flow, $h$, the swept area of turbine, $\frac{1}{4} \pi d^{2}$, must be represented in a blockage ratio conserving manner in order to ensure correct momentum removal from the flow. In a flow with a prescribed upstream velocity, the available power is over-predicted in the depth-averaged simulation when the blockage ratio is too high, as a greater pressure drop may be achieved in the channel for the same thrust as the blockage is increased. Similarly, if the total energy removed from the channel is prescribed (a change in pressure head is given), then there are fewer mixing losses behind the more highly blocked, depth-averaged turbines for a given thrust, also leading to an over-prediction of the available power. These dynamics are described in further detail in Section 3.

One approach to address these challenges in depth-averaged simulations is to model the turbine array in aggregate, rather than as individual turbines. This approach allows the flow speed through the array to be determined in the numerical simulation, and an actuator disk model is applied, as detailed below, to determine the corresponding flow speed through the turbines that is consistent with the thrust applied to the flow. Furthermore, the actuator disk model ensures the blockage ratio in the depth-averaged simulation is consistent with the three-dimensional array.

\section{Separation of Scales}

Figure 1 illustrates the separation of array and device scales in a channel with inlet velocity $u_{\infty}$, where energy extraction can be separated into several stages. The total (global) power removed from the channel, $P_{g}$, is the sum of the power available to the array, $P_{a}$, and dissipated in the array wake, $P_{a, m i x}$. Likewise, the power at the device-scale is the sum of the available device power, $P_{d}$, and power dissipated in device-scale mixing $P_{d, m i x}$. We assume that all turbines operate identically and thus neglect fence end effects, which [10] show to be an appropriate approximation for fences longer than eight turbines. Thus, each actuator disk exerts a thrust $T_{d}$ on the flow, which summed over the $n$ turbines in the array gives the array thrust, $T_{a}$. The velocity through the array is $u_{a}$, and $u_{d}$ through each device, allowing the power removed at the channel, array, and device scales to be written:

$$
P_{g}=T_{a} u_{\infty}=P_{a}+P_{a, m i x}, \quad P_{a}=T_{a} u_{a}=n P_{d}+n P_{d, m i x}, \quad \text { and } \quad P_{d}=T_{d} u_{d} .
$$

Mixing losses represent energy transfer from the mean flow to turbulent mixing processes, the result of shearing between the core and bypass flows due to differing flows speeds. Although these losses cannot be converted into useful power, they must be accounted for as they affect the overall tidal resource and hence accurate estimation of the available array power. 


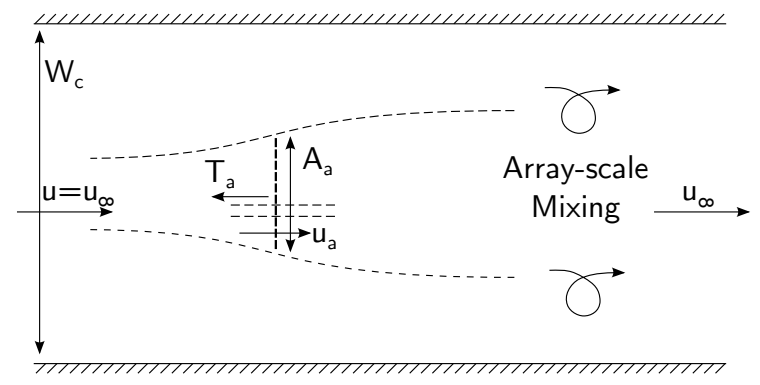

(a) Array-scale flow around a turbine array.

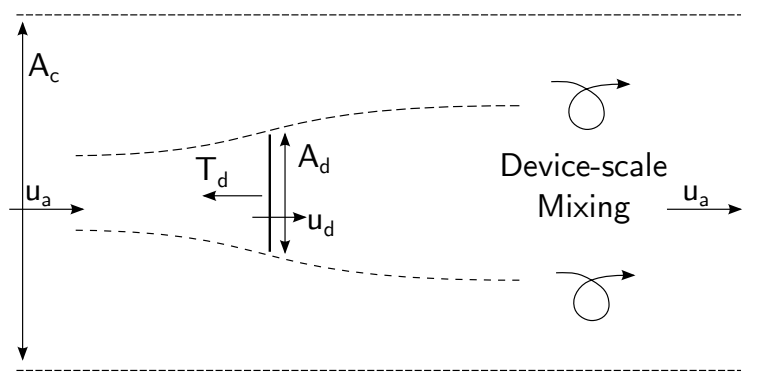

(b) Device-scale flow around a single turbine.

Figure 1: Sketches of the multi-scale flow in a tidal array: a) an array of tidal turbines partially spanning a wide channel, and b) a single turbine and surrounding flow passage inside the array. The array velocity $u_{a}$ provides the upstream boundary condition to the device-scale model.

The fraction of the power removed from a channel available to a turbine is termed the 'basin efficiency' [9]. Three efficiency ratios may be defined in the multi-scale framework: the local efficiency of the device, $\eta_{d}$; array efficiency, $\eta_{a}$; and global efficiency, $\eta_{g}$, where the global efficiency is basin efficiency as defined in the literature:

$$
\eta_{d}=\frac{n P_{d}}{P_{a}}, \quad \eta_{a}=\frac{P_{a}}{P_{g}}, \quad \text { and } \quad \eta_{g}=\eta_{d} \eta_{a}=\frac{n P_{d}}{P_{g}} .
$$

Efficiency is bounded between zero and one, where zero corresponds to none of the power removed at that scale being converted into device or array power, and one corresponds to all the power removed at that scale converted into available power. The latter is achieved when the channel cross-section is completely occupied by tidal devices, forcing all the flow through the turbines so there are no bypass flows and therefore no mixing losses at any scale. The efficiencies must lie somewhere between these extremes as the velocity differences in the bypass and core flows cause shearing between the two streams, resulting in energy dissipation through wake mixing. For instance, an unconstrained actuator disk operating at the Lanchester-Betz limit has $\eta_{d}=\frac{2}{3}$, i.e., $\frac{1}{3}$ of the energy removed from the flow is lost in downstream wake mixing.

\subsection{Matching the array and device scales}

The array- and device-scale problems may be analysed quasi-independently, subject to the thrust relationship $T_{a}=n T_{d}$. A similar relationship does not hold between the array and device power as array power also includes power dissipation in the device wakes, so $P_{a} \neq n P_{d}$. Figure 2 compares depth-averaged simulations specifying array thrust and power with a 3D RANS simulation of eight $20 \mathrm{~m}$ diameter, $d$, actuator disks by Nishino and Willden [10] with uniform channel inflow speed $2 \mathrm{~ms}^{-1}$ and depth $2 d$. The power available to the 3D RANS array is found to be $8.8 \mathrm{MW}$, applying a total thrust of $7.3 \mathrm{MN}$.

A depth-averaged array extracting $8.8 \mathrm{MW}$ at the array scale, $P_{a}=9.9 \mathrm{MW}$, shown in Figure 2(b), requires $5.5 \mathrm{MN}$ of thrust. The thrust underestimation comes from the erroneous assumption that the flow speed through the array is the same as that through the actuator disks and therefore the total energy flux through the array is available to the disks. This does not account for the bypass flow around the disks which limits the available power, thus underestimating the thrust required to achieve the desired realisable power level. The same total thrust as found from the 3D simulation is applied as in Figure 2(c), resulting in an array power, $P_{a}$, of 11MW. This could be erroneously interpreted as the realisable power, which would provide an overestimate as the flow speed through the array and disks is simulated to be the same, not accounting for device-scale bypass and corresponding mass flow reduction through the disks, which would limit the realisable power to be less than $P_{a}$.

The mismatch between power and thrust in two- and three-dimensional simulations arises because of the unresolved flow around the device. Implementations of actuator disk arrays in two-dimensional simulations may be summarised in three categories: 


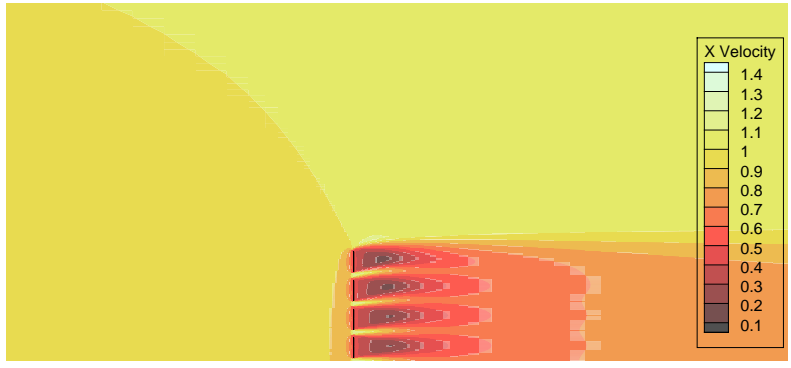

(a) Hub-height centre plane streamwise velocity contours from three-dimensional RANS actuator disk simulation, [10].

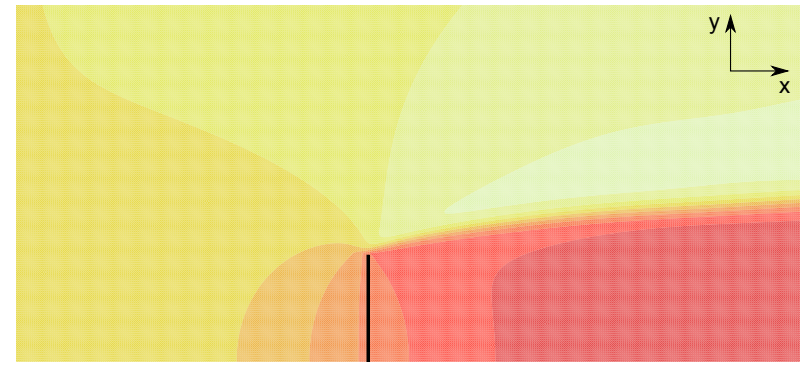

(b) Depth-averaged streamwise velocity contours from a SWE simulation matching total power extracted by array, $P_{a}$, to the total available power simulated in (a), $n P_{d}$.

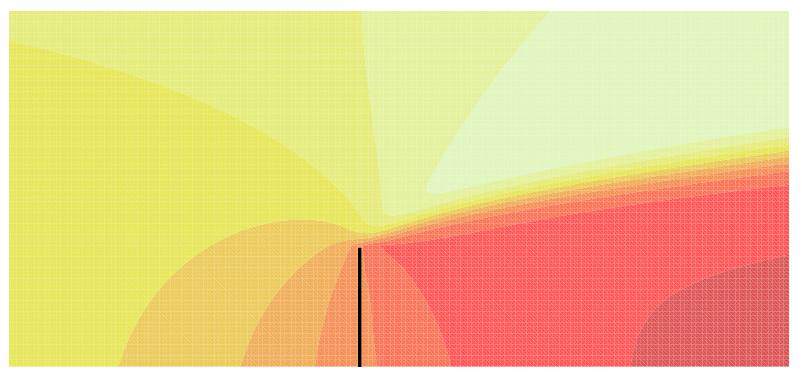

(c) Depth-averaged streamwise velocity contours from a SWE simulation matching the thrust applied by array, $T_{a}$, to the total array thrust simulated in (a), $n T_{d}$.

Figure 2: Comparison between a horizontal plane of the normalised streamwise velocity contours at (a) hub-height in a three dimensional actuator disk simulation and, (b) and (c), two depth averaged simulations matching the power and thrust coefficients of the turbine array. The array/turbine positions are indicated with a black line, with a symmetry plane around the lower boundary of the images. The flow is from left to right.

1. Matching depth-averaged actuator disk thrust to the array thrust, which over-predicts the available power through $P_{a}=T_{a} u_{a}$, as $u_{a}>u_{d}$, but correctly simulates momentum removal from the flow,

2. Matching desired depth-averaged available power to the array power, i.e. setting $P_{a}=n P_{d}$, which under-estimates the required thrust, thus incorrectly estimating momentum removal from the flow and consequent far-field changes,

3. Matching thrust as in (1) so momentum removal is correctly simulated, and modelling the device-scale flow to determine the available power for a given applied array thrust.

The only fully correct approach is (3). A correction is required to calculate the available power from the applied thrust, requiring modelling of the device-scale flow. The array power, $P_{a}$ is often known in a depthaveraged simulation, therefore the array efficiency, $\eta_{a}$, defined in Equation (2) is known as $P_{g}=T_{a} u_{\infty}$ is also known as array thrust is prescribed. The device power, $P_{d}$, must be determined in order to find the maximum power available to idealised devices in order to implement method (3).

Devices cannot be directly resolved within depth-averaged simulations in a numerically stable and blockage ratio-preserving way. One method to determine the available power is to use the definitions in Equation (2) to determine $P_{d}$ through the local efficiency, $\eta_{d}$, of the tidal devices, determined analytically. The correction, multiplying $\eta_{d}$ by the array power, $P_{a}$, shall be employed, where $\eta_{d}$ is derived for a given device thrust based on the analytic solution of Garrett and Cummins [5] for flow around an actuator disk.

\section{Analytical Solution}

Analytic solutions for the power of actuator disks in a rigid lid tidal channel have been derived by Garrett and Cummins [5], and for disks in a channel with a deformable free surface by Whelan et al. [13] and Vogel 
Table 1: Non-dimensionalised variables

\begin{tabular}{|c|c|c|}
\hline Dimensional & Non-dimensional & Definition \\
\hline Core velocities & $\alpha$ & $\alpha=u_{d} / u_{a}$ \\
\hline Bypass velocities & $\beta$ & $\beta=u_{b} / u_{a}$ \\
\hline Area & $B_{L}$ & $B_{L}=A_{d} / A_{c}$ \\
\hline Thrust & $C_{T L}$ & $C_{T L}=T_{d} /\left(\frac{1}{2} \rho u_{1}^{2} A_{d}\right)$ \\
\hline Power & $C_{P L}$ & $C_{P L}=P_{d} /\left(\frac{1}{2} \rho u_{1}^{3} A_{d}\right)$ \\
\hline
\end{tabular}

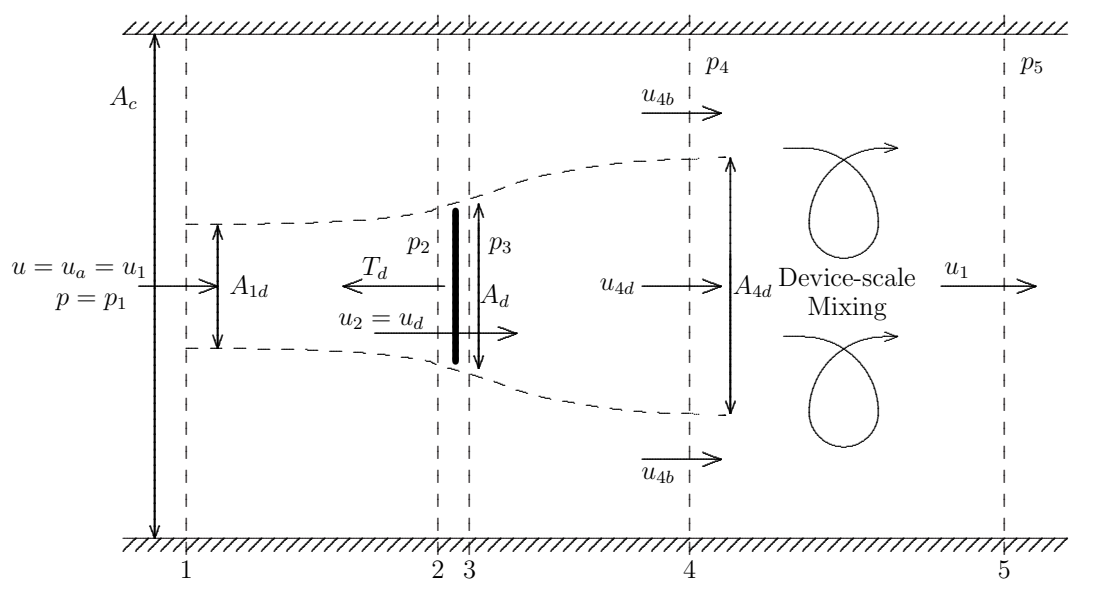

Figure 3: Cross-section of the flow field around an actuator disk in a channel with a rigid lid. Adapted from [5].

et al. [12]. An analytic solution for $\eta_{d}$ will be derived in both the rigid lid and open channel frameworks.

Solutions for $\eta_{d}$ in the rigid lid and free surface models will be presented using the non-dimensionalised variables defined in Table 1. Velocities $\alpha$ and $\beta$ are non-dimensionalised in terms of the upstream devicescale velocity, $u_{a}$. The frontal area of the actuator disk, $A_{d}$, is normalised by the flow passage cross-sectional area, $A_{c}$, for the local blockage, $B_{L}$. Device thrust, $T_{d}$, is normalised against the upstream dynamic pressure to give the thrust coefficient, $C_{T L}$, and the power, $P_{d}$, is normalised by the upstream flux of kinetic energy for the power coefficient, $C_{P L}$.

\subsection{Rigid lid}

Figure 3 shows an actuator disk in a channel with a rigid lid, adapted from Garrett and Cummins [5]. Three regions are important: the core flow, that which passes through the disk, between stations one and four; the bypass flow, that which does not flow through the disk, between stations one and four; and the mixing region between stations four and five. Station four is located where the hydrostatic pressure in the core and bypass flows equalises, although a velocity difference still exists between the two flows. Energy losses associated with shearing of the core and bypass flows are assumed only to occur in the mixing region, so that the total power removed from the device-scale flow is the sum of the available power, $P_{d}$, and the power dissipated in the mixing zone, $P_{d, m i x}$, as defined in Equation (1). The power available to the device is related to the disk thrust, $T_{d}$, and the velocity $u_{2}$, i.e. $P_{d}=T_{d} u_{2}$. Garrett and Cummins' rigid lid momentum analysis will be adapted to determine $u_{2}$, and therefore the available power, given a specified thrust.

Defining the force on the actuator disk as $T_{d}=A_{d}\left(p_{2}-p_{3}\right)$, the Bernoulli equation in the bypass flow between stations one and four, and for the core flow between one and two, and then between three and four, can be used to express the device thrust as a function of the core and bypass velocities at station four:

$$
T_{d}=\frac{1}{2} \rho A_{d}\left(u_{4 b}^{2}-u_{4 d}^{2}\right) .
$$


Continuity between stations one and four requires:

$$
A_{4 d}\left(u_{4 b}-u_{4 d}\right)=A_{c}\left(u_{4 b}-u_{1}\right),
$$

Non-dimensionalising as in Table 1 and using the continuity relation above, allows conservation of momentum between stations one and four to be expressed as:

$$
\frac{A_{d}}{A_{4 d}} C_{T L}=\left(\beta_{4}-\alpha_{4}\right)\left(\beta_{4}+2 \alpha_{4}-1\right)
$$

Non-dimensionalised, Equation (3) can be rearranged for the bypass velocity, $\beta_{4}$. Equations (3) and (5), recognising that $u_{4 d} A_{4 d}=A_{d} u_{2}$, are combined to express $\alpha_{2}$ in terms of $\alpha_{4}$ and $\beta_{4}$ :

$$
\beta_{4}=\sqrt{C_{T L}+\alpha_{4}^{2}}, \quad \text { and } \quad \alpha_{2}=\frac{\alpha_{4}\left(\beta_{4}+\alpha_{4}\right)}{\beta_{4}+2 \alpha_{4}-1} .
$$

$\alpha_{4}$ is expressed in terms of $C_{T L}$ by substituting $\beta_{4}$ into Equation (6):

$$
C_{T L}(1-B)+\left(\alpha_{4}-1\right)^{2}+2 \sqrt{C_{T L}+\alpha_{4}^{2}}\left(\alpha_{4}-1\right)=0 .
$$

For a given geometric blockage, $B$, and device thrust coefficient, $C_{T L}$, available power can be determined by solving Equation (7) for $\alpha_{4}$, determining $\beta_{4}$ and $\alpha_{2}$ using Equation (6), and finally solving the power coefficient $C_{P L}$ as:

$$
C_{P L}=\alpha_{2} C_{T L}
$$

The total power is the sum of available power and the power dissipated in wake mixing. The power dissipated in the wake is found with a momentum balance between stations four and five:

$$
\left(A_{c}-A_{4 d}\right)\left(p_{4}+\rho u_{4 b}^{2}\right)+A_{4 d}\left(p_{4}+\rho u_{4 d}^{2}\right)=A_{c}\left(p_{5}+\rho u_{1}^{2}\right),
$$

where the flow is assumed fully remixed at five. Equation (4) and (5) may be combined to yield an expression for $C_{T L}$ :

$$
B_{L} C_{T L}=\left(\beta_{4}-1\right)\left(\beta_{4}+2 \alpha_{4}-1\right) .
$$

2 From conservation of momentum between stations one and five, the total power removed from the device-scale flow is $P_{T o t}=T_{d} u_{1}$, so the coefficient for the total power removed from the device scale, $C_{P L, T o t}$, is:

$$
C_{P L, T o t}=\frac{1}{B_{L}}\left(\beta_{4}-1\right)\left(\beta_{4}+2 \alpha_{4}-1\right)=C_{T L} .
$$

Using the definition of $\eta_{d}$ and $C_{P L}$ in Equations in (2) and (8) respective, the local efficiency can be expressed as:

$$
\eta_{d}=\frac{C_{P L}}{C_{P L, T o t}}=\frac{\alpha_{2} C_{T L}}{C_{T L}}=\alpha_{2} .
$$

Figure 4 shows the effect of blockage ratio, $B_{L}$, on local efficiency $\eta_{d}$ against local thrust coefficient $C_{T L}$. Local efficiency decreases more quickly at lower than higher blockage ratios. For the highest blockage, $B_{L}=0.8$, which corresponds to a flow passage measuring one diameter in width and height, a thrust coefficient $C_{T L}=1$ results in an efficiency $\eta_{d}=0.96$. This contrasts sharply with a local efficiency $\eta_{d}=0.65$ at the same thrust coefficient when the blockage ratio is $B_{L}=0.05$, which is a more representative blockage for proposed array designs. This implies greater shear between core and bypass in the lower blockage case for the same level of applied thrust. Recalling that the relationship between available device power and the array power is $P_{d}=\eta_{d} P_{a}$, it is clear that applying a correction to the power extracted from the depth-averaged simulation of a turbine array is particularly important for low blockage arrays.

Local efficiency is negatively correlated with $C_{T L}$ because the flux through the device, $u_{d}$, reduces as $C_{T L}$ increases, accelerating the bypass flow $u_{b}$ to conserve mass flux. Stronger shearing is caused by the increased 


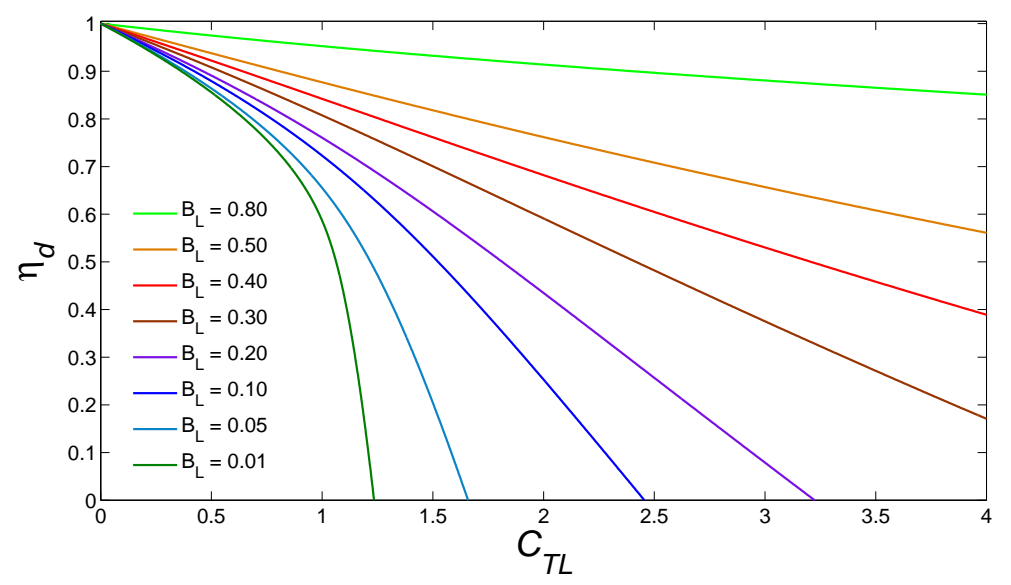

Figure 4: Plot of local efficiency, $\eta_{d}$, against local thrust coefficient, $C_{T L}$, for eight local blockage ratios, $B_{L}$.

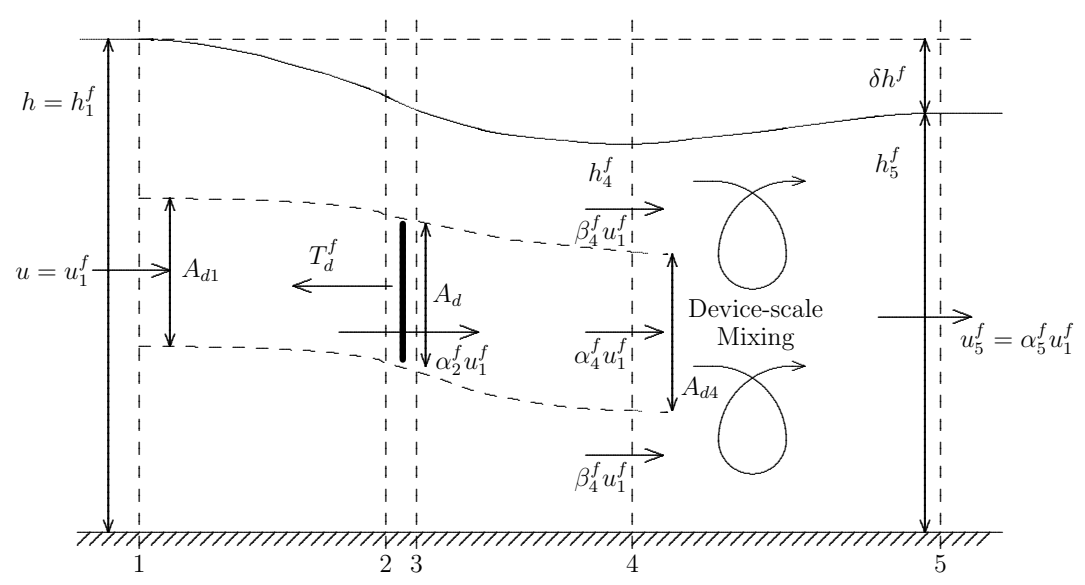

Figure 5: Device-scale flow field around an actuator disk in an open channel. Adapted from Houlsby and Vogel [6].

velocity difference between the core and bypass flow speeds in the wake, thus more energy is dissipated in the mixing region. Local efficiency is particularly heavily penalised in low blockage arrays as $C_{T L}$ increases because the bypass flow is relatively unconstrained and there is little resistance to core flow deceleration and corresponding bypass flow acceleration as $C_{T L}$ increases. Devices operating in relatively constrained flow fields in high blockage arrays experience much greater resistance to deceleration of core flow and hence acceleration of the bypass flow, and consequently $\eta_{d}$ is higher than in low blockage arrays as $C_{T L}$ increases.

\subsection{Deformable free surface}

Energy extraction by the actuator disks results in a gradient in static head developing in the simulated array. Open channel theory predicts the free surface should drop and the flow accelerate to conserve mass flux through the flow passage. A correction for the simulated shallow water flow model can be formulated to take this into account using the theory developed by Whelan et al. [13] and Houlsby and Vogel [6]. The correction matches the change in free surface elevation at the upstream and downstream edges of the array region to the boundary conditions of the device-scale sub-model. The advantage of such a correction is that it may be applied to simulations modelling the array as enhanced bed friction or similar methods that do not directly represent any more of the flow physics other than the thrust applied by the actuator disks.

Figure 5 is a diagram of the flow field around an actuator disk in an open channel, in which variables have a superscript $f$ to distinguish them from the rigid lid model. The thrust, $T_{d}^{f}$, applied by a disk in a flow 
passage with a change in free surface elevation $\delta h^{f}=h_{1}^{f}-h_{5}^{f}$ can be determined by a momentum balance between stations one and five:

$$
\frac{1}{2} \rho g b\left(h_{1}^{f 2}-h_{5}^{f 2}\right)-T_{d}^{f}=\rho b h_{1}^{f} u_{1}^{f}\left(u_{5}^{f}-u_{1}^{f}\right)
$$

where $b$ is the flow passage width, $g$ is gravity, and $u_{5}^{f}$ is the downstream velocity once the core and bypass flows have remixed. Continuity requires:

$$
h_{1}^{f} u_{1}^{f}=h_{5}^{f} u_{5}^{f} .
$$

Substituting the definition for $\delta h^{f}$ and continuity into Equation 13 yields a cubic in $\delta h^{f} / h_{1}^{f}$ :

$$
\frac{1}{2} \rho g b h_{1}^{f 3}\left(2 \frac{\delta h^{f}}{h_{1}^{f}}-\left(\frac{\delta h^{f}}{h_{1}^{f}}\right)^{2}\right)\left(1-\frac{\delta h^{f}}{h_{1}^{f}}\right)-h_{1}^{f} T_{d}^{f}\left(1-\frac{\delta h^{f}}{h_{1}^{f}}\right)=\rho b h_{1}^{f 2} u_{1}^{f 2} \frac{\delta h^{f}}{h_{1}^{f}} .
$$

239 Introducing the Froude number $F r=u_{1}^{f} / \sqrt{g h_{1}^{f}}$, local blockage $B_{L}^{f}=A_{d} / b h_{1}^{f}$, and the local thrust coefficient ${ }_{240} C_{T L}^{f}=T_{d}^{f} / \frac{1}{2} \rho u_{1}^{f 2} A_{d}$, Equation (15) can be rearranged for the local thrust coefficient $C_{T L}^{f}$ :

$$
C_{T L}^{f}=\frac{\frac{\delta h^{f}}{h_{1}^{f}}}{F r^{2} B_{L}^{f}\left(1-\frac{\delta h^{f}}{h_{1}^{f}}\right)}\left(\left(\frac{\delta h^{f}}{h_{1}^{f}}-2\right)\left(\frac{\delta h^{f}}{h_{1}^{f}}-1\right)-2 F r^{2}\right) .
$$

The total power removed from the flow between stations one and five is the sum of the power available to the disk, $P_{d}^{f}$ and the power dissipated in the device-scale wake, $P_{d, m i x}^{f}$ :

$$
P_{d}^{f}+P_{d, m i x}^{f}=\frac{1}{2} \rho u_{1}^{f 3} b h_{1}^{f}-\frac{1}{2} \rho u_{1}^{f 3} b h_{1}^{f}\left(\frac{h_{1}^{f}}{h_{1}^{f}-\delta h^{f}}\right)^{2}+\rho g b h_{1}^{f} u_{1}^{f}\left(h_{1}^{f}-h_{5}^{f}\right) .
$$

${ }_{243}$ Defining a power coefficient for the total power removed at the device scale in an open channel as $C_{P L, T o t}^{f}=$ ${ }^{244} \quad\left(P_{d}^{f}+P_{d, m i x}^{f}\right) / \frac{1}{2} \rho u_{1}^{f 3} A_{d}$, we may therefore write:

$$
C_{P L, T o t}^{f}=\frac{1}{B_{L}^{f}}\left(1-\left(1-\frac{\delta h^{f}}{h_{1}^{f}}\right)^{-2}+\frac{2}{F r^{2}} \frac{\delta h^{f}}{h_{1}^{f}}\right) .
$$

The local efficiency for the free surface model, $\eta_{d}^{f}$, is the ratio of the power available to the actuator disk to the total power removed at the device scale:

$$
\eta_{d}^{f}=\frac{P_{d}^{f}}{P_{d}^{f}+P_{d, m i x}^{f}}=\frac{C_{P L}^{f}}{C_{P L, T o t}^{f}}=\frac{\alpha_{2}^{f} C_{T L}^{f}}{C_{P L, T o t}^{f}} .
$$

247 Substituting the thrust coefficient calculated in Equation (16), and the total power removed from the flow ${ }_{248} C_{P L, T o t}^{f}$, Equation (18), into the efficiency definition yields:

$$
\eta_{d}^{f}=\frac{\alpha_{2}^{f} \frac{\delta h^{f}}{h_{1}^{f}}\left(1-\frac{\delta h^{f}}{h_{1}^{f}}\right)}{F r^{2}} \frac{\left(\frac{\delta h^{f}}{h_{1}^{f}}-2\right)\left(\frac{\delta h^{f}}{h_{1}^{f}}-1\right)-2 F r^{2}}{\left(1-\frac{\delta h^{f}}{h_{1}^{f}}\right)^{2}\left(1+\frac{2}{F r^{2}} \frac{\delta h^{f}}{h_{1}^{f}}\right)-1} .
$$

The upstream Froude number and the relative change in free surface elevation, $\delta h^{f} / h_{1}^{f}$, are inputs from the depth-averaged simulation, leaving $\alpha_{2}^{f}$ as the remaining quantity required to enable the efficiency, $\eta_{d}^{f}$, and 
hence available power $P_{d}^{f}$ to be evaluated. However, this does not present a problem as the induction factor, $\alpha_{2}^{f}$, is completely determined by solving LMADT for an actuator disk in an open channel for a given $\delta h^{f} / h_{1}^{f}$ ratio, found following Houlsby and Vogel [6]. For the disk LMADT model it is mathematically convenient to begin by specifying the wake induction factor, $\alpha_{4}^{f}$, and then solving for $\alpha_{2}^{f}$ and $\delta h^{f} / h_{1}^{f}$, as summarised below. It follows from conservation of linear momentum from stations one to four that the bypass velocity $\beta_{4}^{f}$ is found by solving the quartic:

$$
\begin{aligned}
\frac{1}{2} F r^{2} \beta_{4}^{f 4}+2 \alpha_{4}^{f} F r^{2} \beta_{4}^{f 3}-\left(2-2 B_{L}^{f}+F r^{2}\right) \beta_{4}^{f 2}- & \left(4 \alpha_{4}^{f}+2 \alpha_{4}^{f} F r^{2}-4\right) \beta_{4}^{f} \\
& +\left(\frac{1}{2} F r^{2}+4 \alpha_{4}^{f}-2 B_{L}^{f} \alpha_{4}^{f 2}-2\right)=0 .
\end{aligned}
$$

The induction factor $\alpha_{2}^{f}$ can be found as a function of $\alpha_{4}^{f}$ and $\beta_{4}^{f}$ :

$$
\alpha_{2}^{f}=\frac{\alpha_{4}^{f} \beta_{4}^{f}}{B_{L}^{f}\left(\beta_{4}^{f}-\alpha_{4}^{f}\right)}\left(1-\frac{1}{2} F r^{2}\left(\beta_{4}^{f^{2}}-1\right)-\frac{1}{\beta_{4}^{f}}\right) .
$$

Using the Bernoulli equation in the core streamtube upstream and downstream of the disk, together with energy conservation in the bypass flow (ahead of station four in both cases), allows the device thrust to be written as a local thrust coefficient expressed as a function of $\alpha_{4}^{f}$ and $\beta_{4}^{f}$ :

$$
C_{T L}^{f}=\beta_{4}^{f 2}-\alpha_{4}^{f 2}
$$

Using this expression to replace the thrust coefficient in the cubic for $\delta h^{f} / h_{1}^{f}$ derived from the momentum balance over the device scale in Equation (16) results in a non-dimensional cubic equation for $\delta h^{f} / h_{1}^{f}$ which, using Equation (21) to relate $\beta_{4}^{f}$ to $\alpha_{4}^{f}$, may be solved in terms of $\alpha_{4}^{f}$ :

$$
\left(\frac{\delta h^{f}}{h_{1}^{f}}\right)^{3}-3\left(\frac{\delta h^{f}}{h_{1}^{f}}\right)^{2}+\left(2+F r^{2}\left(B_{L}^{f}\left(\beta_{4}^{f 2}-\alpha_{4}^{f 2}\right)-2\right)\right) \frac{\delta h^{f}}{h_{1}^{f}}-F r^{2} B_{L}^{f}\left(\beta_{4}^{f 2}-\alpha_{4}^{f 2}\right)=0 .
$$

Equations (22) for $\alpha_{2}^{f}$ and (24) for $\delta h^{f} / h_{1}^{f}$ can be solved for the physically possible range of $\alpha_{4}^{f}$ values (0 to 1$)$ so that $\alpha_{2}^{f}$ can be effectively related directly $\delta h_{f} / h_{1}^{f}$ and hence $\alpha_{2}^{f}$ can be set by the measured $\delta h^{f} / h_{1}^{f}$ from the shallow water simulation. Although this method more accurately represents the physics of the devicescale flow, it is very sensitive to the location of the upstream and downstream boundaries of the region in which the array is simulated, as the change in free surface elevation is calculated from these points. It is assumed that over the local scale energy removal by the devices dominates energy losses due to bed friction and ambient turbulence, as these will also contribute to the change in free surface elevation.

Figure 6 shows the effect that free surface deformation has on $\eta_{d}^{f}$ in comparison to the predictions of the rigid lid model. The Froude number, $F r=0.2$, is chosen to be representative of sites under investigation for tidal array development. Local efficiency is uniformly improved across all blockage ratios in the free surface model, the magnitude of the efficiency enhancement increasing with blockage ratio because $\alpha_{2}$ is higher in the free surface model. The cross sectional area of the static head equalisation plane (4) in the free surface model is smaller than the pressure equalisation plane (4) in the rigid lid model due to the free surface deformation in response to the lower hydrostatic pressure. Hence the flow accelerates in the free surface model in order to conserve mass flux through the device-scale, resulting in higher disk velocity, $\alpha_{2}$, and therefore local efficiency, $\eta_{d}$

Although the free surface model more accurately represents the physics of the device-scale flow, differences between it and the rigid lid model are most significant at high local blockage ratios, much greater than those being considered for commercial arrays. There is good agreement between the two models at low blockage ratios and thrust coefficients, and the rigid lid model will generally provide a more tractable solution for the local efficiency. 


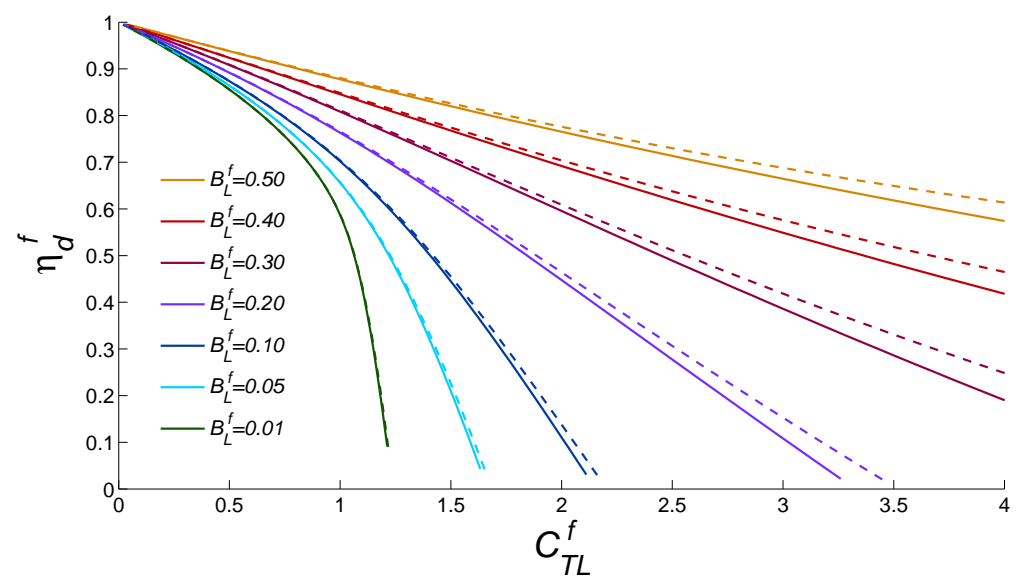

Figure 6: Variation in local efficiency, $\eta_{d}^{f}$, with local thrust coefficient, $C_{T L}$, for seven blockage ratios $B_{L}^{f}$, as predicted by the free surface model, with $F r=0.2$, (dashed lines), and the rigid lid model (solid lines).

\section{Numerical Implementation}

Grid resolution constraints, particularly in coastal and continental shelf scale simulations, mean that the size of cells in a numerical simulation are often much larger than the size of the tidal devices being simulated. In addition to this, it may be numerically necessary to smear the turbine region over multiple adjacent cells in the streamwise direction in order to achieve a numerically stable solution, further abstracting the simulation from the device-scale flow field.

The total power removed from the device-scale flow is the sum of the available power and the power dissipated by device-scale mixing, and is equal to the array power, $P_{a}$, in Equation (1). The thrust applied by the array is the sum of the thrust applied by the turbines in the array, $n T_{d}$, if uniform thrust is applied. Thus, if device thrust is known, the array thrust can be specified as a simulation parameter. The array power, $P_{a}=u_{a} T_{a}$, can be determined from the simulations, and the analytic correction that yields $\eta_{d}$ applied during post-processing to determine the available device power, as $P_{d}=\eta_{d} P_{a} / n$.

A number of techniques exist to discretise depth-averaged simulations. The DG-FEM approach permits step discontinuities in the fluid depth and velocity across the boundary of adjacent elements, which may be used if one wishes to reduce the entire device-scale model to occur over the interface between two adjacent elements (see, for example [3]). The flow depth and velocity in the upstream cell provide the upstream boundary conditions to the device-scale model and are used to calculate the depth and velocity in the element downstream of the array.

CG-FEM is more widely used to discretise depth-averaged simulations, but does not permit step discontinuities in the depth and velocity between adjacent elements. It is therefore necessary to 'smear' the array in the streamwise direction of the flow to avoid numerical instabilities arising from the application of the array thrust. The total power extracted by a smeared array in a CG-FEM scheme is:

$$
P_{a}=\sum_{i=1}^{N_{\Omega_{a}}} T_{i} u_{i}
$$

where $N_{\Omega_{a}}$ is the number of elements in the region $\Omega_{a}$ over which the array thrust is smeared, and $T_{i}$ is the thrust and $u_{i}$ the streamwise velocity at position $i$. The local efficiency multiplies the array power $P_{a}$ to determine the total power available to the turbine array. 


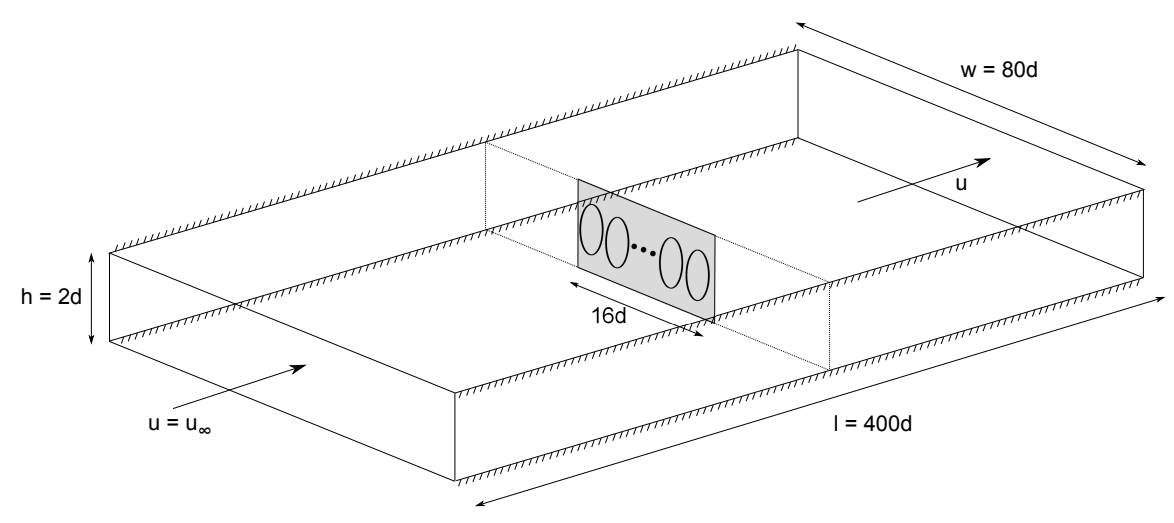

Figure 7: Diagram of numerical flume for comparison between 3D RANS and depth-averaged simulations. Turbines are explicitly simulated in the 3D RANS computations, and represented by a region of increased resistance (shown in grey) in the depthaveraged computations. Inter-turbine spacing in the array is $1 d$.

\section{Results}

\section{Comparison to 3D RANS Simulation}

The rigid lid analytic sub-model is applied to a depth-averaged simulation of an array of eight $20 \mathrm{~m}$ diameter actuator disks with an inter-turbine cross-stream spacing of 1d in a channel of width $80 d$ with an inflow speed of $2 \mathrm{~ms}^{-1}$ and depth of $2 \mathrm{~d}$, as shown in Figure 7 . The frictionless channel is $400 d$ in length, and had a uniform inflow profile in the 3D RANS simulations. The array is modelled as a single area of increased resistance (due to the thrust applied by the array) with a cross-stream width of $16 \mathrm{~d}$ in TELEMAC-2D, an open source two-dimensional CG-FEM code. The simulation is designed to be the depth-averaged equivalent of a three dimensional RANS simulation for eight actuator disks by [10], with an overall global blockage ratio (total frontal area of actuator disks normalised on channel cross-sectional area) $B_{G}=n A_{d} / A_{c}=0.04$. The thrust-power curve is known for the three dimensional simulation, and provides an array performance benchmark which includes all flow bypasses and remixing.

To aid comparison between the 3D RANS and depth-averaged simulations, slip wall conditions were used on the upper, lower, and lateral boundaries of the domain in order to preserve the uniform flow profile through the domain. The turbulence intensity at the inlet was very low, c. $0.1 \%$, in order to reduce the impact of the differences between two- and three-dimensional implementations of the $k-\epsilon$ turbulence model (with coefficients following [10]) in the depth-averaged and 3D RANS simulations respectively. Consequently, the flow in the domains matched well in the absence of the array, with small differences in the solutions due to the more dissipative nature of the depth-averaged model. It should be noted that turbulence intensity increases in the wake downstream of the array, with differences arising between the two- and three-dimensional models due to the effect of shear around the edges of the actuator disks, which could be accounted for in the shallow water model with an induced turbulence model in a similar manner to that proposed by [8]. However, this is was not considered in the present study.

Figure 8 shows the global thrust coefficient $C_{T G}=n T_{d} / \frac{1}{2} \rho A_{c} u_{\infty}^{2}$ vs. global power coefficient $C_{P G}=$ $P_{G} / \frac{1}{2} \rho A_{c} u_{\infty}^{3}$ curve of the 3D RANS simulation, compared to two analytic models and two shallow water simulations of the same channel. The array is modelled using open channel LMADT [6] with $\mathrm{Fr}=0.20$, which assumes power extraction is governed only by the ratio of the channel cross-section occupied by the array. This theoretical power curve, with peak power coefficient $C_{P G, \max }=0.93$, over predicts the $2 \mathrm{D}$ depth averaged simulated power curve for the same assumption $\left(C_{P G, \max }=0.85\right)$ because of the influence of viscous mixing effects and the cross-stream flux out of the simulated array region is neglected. These results indicate that it is insufficient to solely consider the array blockage as the area ratio governing the power available to an array, and therefore device-scale effects must also be considered.

Device-scale blockage may be considered alongside the array-scale blockage of the channel, as presented in the analytic partial array model of Vogel et al. [12]. The peak power decreases to $C_{P G, \max }=0.79$ because of the 


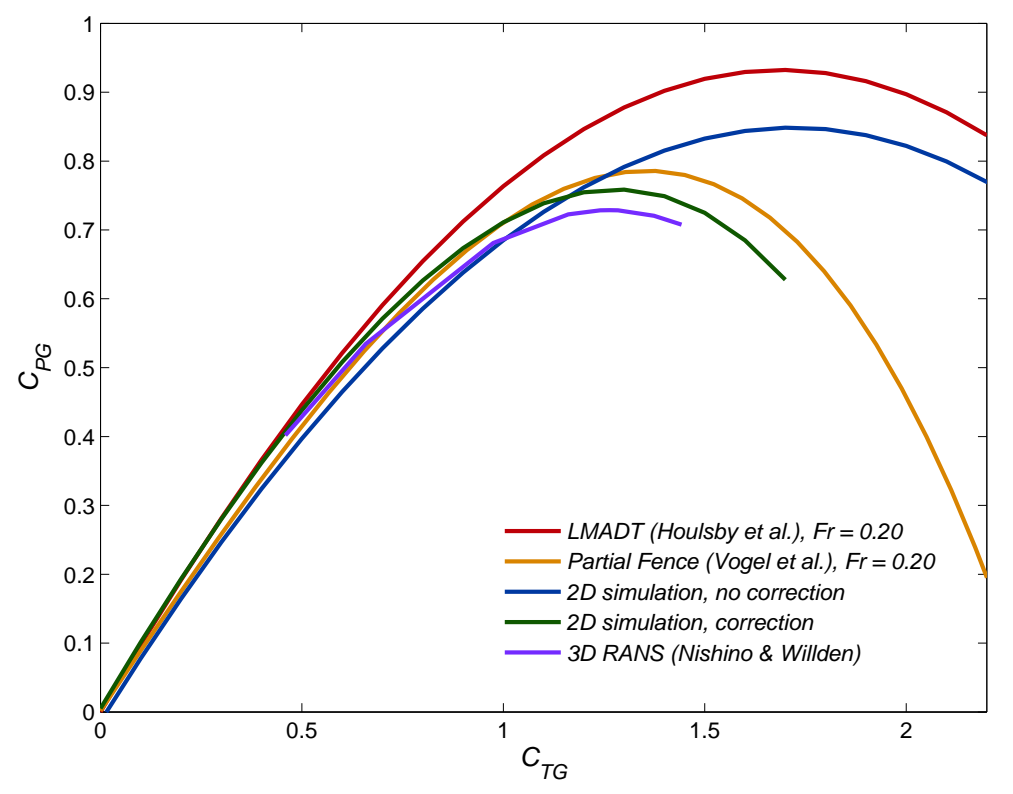

Figure 8: Comparison of the power curve $C_{P G}$ from 3D RANS simulations (purple) of Nishino and Willden [10], to the power curve predicted by LMADT (red), a depth-averaged array without the analytical correction (dark blue), two-scale analytical model (orange), and a depth-averaged array with analytical correction applied (green).

device-scale bypass, which reduces the available device power. In the corrected depth-averaged simulation $C_{P G, \max }=0.76$, which occurs at a lower thrust than the uncorrected simulation when device-scale flow is included because the reduction in flow speed through the disk due to the device thrust is compounded by the reduction in the flow speed through the array due to the array thrust. Thus, the available power is reduced, especially at higher thrusts, and hence peak power occurs at lower thrust. The two scale analytic model predicts a slightly higher peak power than the corrected depth-averaged simulation because the analytic model assumes that the flow is inviscid. The corrected depth-averaged simulation compares well to the 3D RANS power curve, which has a peak power coefficient of $C_{P G, \max }=0.73$, a difference of $4 \%$ between the two simulations.

It is often of interest to determine the thrust required to achieve a specified power output. It has been demonstrated that the available power is less than the power implied by the product of the array thrust and the bulk flow speed through the array. Indeed, a higher device thrust is required to achieve the specified available power once the analytic correction has been applied. The required thrust can be found by determining $\eta_{d}$ vs. $C_{T L}$ and $\eta_{d}$ vs. $C_{P L}$, given the local blockage ratio, and matching to finding the thrust to be applied to the flow required to achieve the desired available power. It may not necessarily be possible to find a solution, particularly at low blockages, where the flow can divert into the large, relatively unconstrained device bypass rather than go through the device as the thrust is increased, limiting the power that may be achieved without increasing the frontal area or number of turbines.

A higher flow rate through the disk can be maintained at higher blockage ratios due to the greater confinement of the flow, resulting in improved power as blockage increases. This also reduces the velocity difference in the bypass for a given thrust level, in turn reducing the shearing between the core and bypass flows, which reduces loses and improves $\eta_{d}$. Very high blockage arrays can have a local efficiency close to $\eta_{d}=1$ over the range of global thrust coefficients that turbine arrays may operate at, and therefore uncorrected depthaveraged simulations predict a total available power which is not too much higher than the power potential of the array. However, uncorrected depth-averaged simulations of low blockage arrays, for which $\eta_{d} \lesssim 0.6$, may over predict the available power by $40 \%$ or more which significantly misrepresents the power potential of the arrays, and therefore the analytical correction needs to be applied for more robust yield assessment. 


\begin{tabular}{|c|c||ccc|}
\hline$N_{\text {turbs }}$ & $B_{G} \backslash^{B_{L}}$ & $\frac{\pi}{10}$ & $\frac{\pi}{16}$ & $\frac{\pi}{32}$ \\
\hline \hline 4 & 0.0196 & 0.0624 & 0.0998 & 0.1996 \\
\hline 8 & 0.0393 & 0.1251 & 0.2002 & 0.4003 \\
\hline 16 & 0.0785 & 0.2499 & 0.3998 & 0.7669 \\
\hline 32 & 0.1575 & 0.5013 & 0.8021 & 1.0000 \\
\hline
\end{tabular}

Table 2: Table of array blockage ratio, $B_{A}=B_{G} / B_{L}=A_{a} / W_{c} h$, corresponding to the global, $B_{G}$, and local blockage, $B_{L}$, configurations for comparison of numerical results to analytic simulation.

\section{Comparison to Analytic Model}

Corrected depth-averaged simulations were compared to the analytic partial array model of Nishino and Willden [9] for arrays of different widths in a frictionless rectangular channel 1600m wide and 40m deep with a uniform inflow speed of $2 \mathrm{~ms}^{-1}$. A total of twelve scenarios were considered, with three different local blockage ratios, and four different global blockage ratios. The local blockage ratios were $B_{L}=\frac{\pi}{10} \approx 0.32$, $B_{L}=\frac{\pi}{16} \approx 0.2$, and $B_{L}=\frac{\pi}{32} \approx 0.1$. The global blockages corresponded to arrays composed of four, eight, sixteen and thirty-two devices, as summarised in Table 2. Corrected depth-averaged simulations are compared to analytic solutions for the same local and global blockage ratios. The key modelling differences between the analytic model and the corrected depth-averaged simulations is that the partial array analytic model does not account for cross-stream flow variations, and assumes that the flow presented to the turbines is uniform across the array.

The effect of the number of devices on the $C_{T G}$ vs. $C_{P G}$ relationship for a fixed local blockage ratio of $B_{L}=\frac{\pi}{16}$ is shown for corrected depth-averaged and analytic results in Figure 9. Overall, as the number of devices increases, both the analytic partial array model and the corrected depth-averaged simulations predict that the peak $C_{P G}$ increases. This increase is due to the increase in global blockage, meaning that a greater proportion of the channel is occupied by the turbines as shown by the increase in the array blockage, see Table 2. A greater peak thrust can be achieved due to the increased pressure (static head) difference that may develop in the channel and therefore the peak power increases.

The relative difference in the peak power coefficient determined from the analytic model and corrected depthaveraged simulations reduces as the global blockage increases because the assumption of uniform flow being presented to the devices in the array becomes a better approximation to the flow in the depth-averaged simulation as the number of turbines increases. Devices near the ends of the array (outboard) experience a cross-stream component of flow towards the array ends due to the lower resistance to the flow in the array bypass as compared to the flow passing through the inboard sections of the array. If the device thrust is uniform across the array, this skewed flow past the outboard devices results in a reduced axial flow through the devices, reducing their power, as compared to inboard devices. Conversely, the resistance to flow bypass around the inboard devices is approximately uniform on both sides of the devices, and hence they do not suffer the same reduction in power due to skewed flow as outboard devices.

The effect of array-scale flow diversion around outboard turbines is more significant in shorter arrays where a greater proportion of the devices are affected by the array-end flow diversion. The relative difference between the analytic and simulated results decreases from approximately $20 \%$ for an array of four turbines to approximately $5 \%$ for 32 turbines, where the remaining differences can be attributed to remaining localised end effects, viscous effects and other modelling differences between the idealised analytic solution and the depth-averaged simulation.

The effect of local blockage ratio on the thrust vs. power coefficient relationship is shown in Figure 10 for different numbers of turbines, and therefore fixed global blockage in each sub-plot. The peak power coefficients increase as the number of turbines in the array increases, and agreement between the corrected depth-averaged simulations and analytic solutions is improved for all local blockage ratios as the number of turbines in the array increases, due to the reducing importance of end effects as explained previously. For any given number of devices, the peak power coefficient is achieved at the highest local blockage ratio, $B_{L}=\frac{\pi}{10}$, because at the device scale the disks are able to apply a greater thrust. This is because a greater pressure 


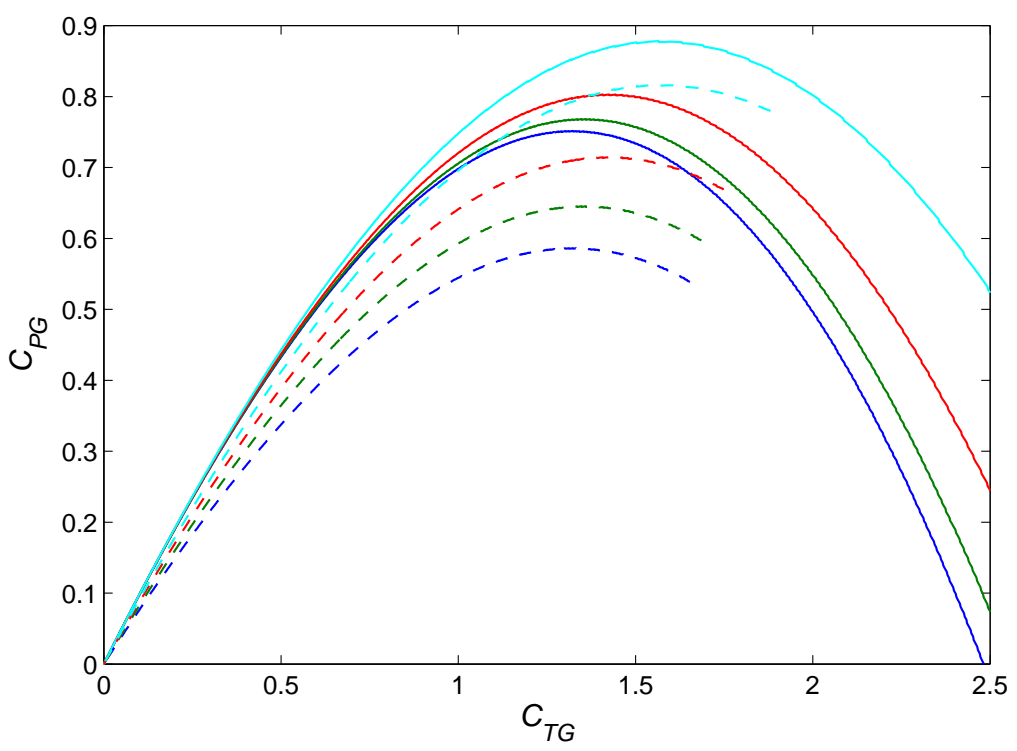

Figure 9: Comparison between corrected depth-averaged simulated (dashed lines) and analytically predicted (solid lines) power coefficient $C_{P G}$ vs. thrust coefficient $C_{T G}$ for constant local blockage $B_{L}=0.2$ for arrays of four (blue), eight (green), sixteen (red), and thirty-two (cyan) turbines.

(static head) difference can be established across the devices as a result of the reduced cross-sectional area of the device-scale bypass and increased implied device bypass resistance. This improves the local efficiency for a given thrust coefficient, which in turn improves the array performance.

Figure 10 shows that the relative difference between the corrected depth-averaged simulations and the analytic solutions is greater at higher local blockage ratios and reduced at lower local blockage ratios for arrays of any length. Devices at higher local blockage ratios apply a greater thrust to the flow which results in a more significant cross-stream variation in flow speed that arises due to the finite length effect of flow diversion past the simulated arrays. Devices in lower local blockage arrays do not benefit to the same extent from the mutual interactions which increase available power in higher local blockage arrays, as the greater inter-turbine spacing provides less resistance to the device-scale flow bypass around the disks. Lower blockage devices must therefore apply a smaller thrust to the flow. The lower local blockage devices are therefore less affected by the decrease in power experienced by the outboard devices of the array than higher local blockage devices, and therefore relative agreement with the analytic solution is improved, albeit for a lower peak power coefficient.

Agreement between the corrected depth-averaged simulations and analytic theory improves substantially as an array is increased from four to eight disks, and then from eight to sixteen disks. The improvement is subject to diminishing returns as the array length increases and the relative influence of the array end effects reduces. The results are largely in agreement with those of Nishino and Willden [10], and indicate that analytic theory and numerical simulation give fairly good agreement for arrays of at least eight devices. The agreement is affected by the local device blockage, and higher blockage arrays generally need to be longer in order to reduce the impact of the array end effects on the overall array power to improve agreement with analytic models. However, the reduction in power of the outboard devices is a necessary consequence of finite length arrays, and therefore merits further attention to reduce the impact it has on the total array power.

\section{Conclusions}

Depth-averaged simulations are an expedient alternative solution to the expensive computational domains required to simulate large tidal turbine arrays in three-dimensions. Several challenges exist for depth-averaged 


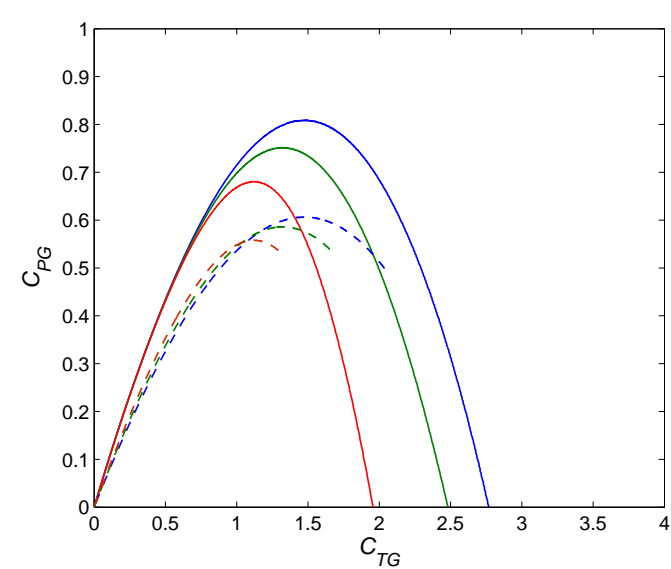

(a) Four turbine array.

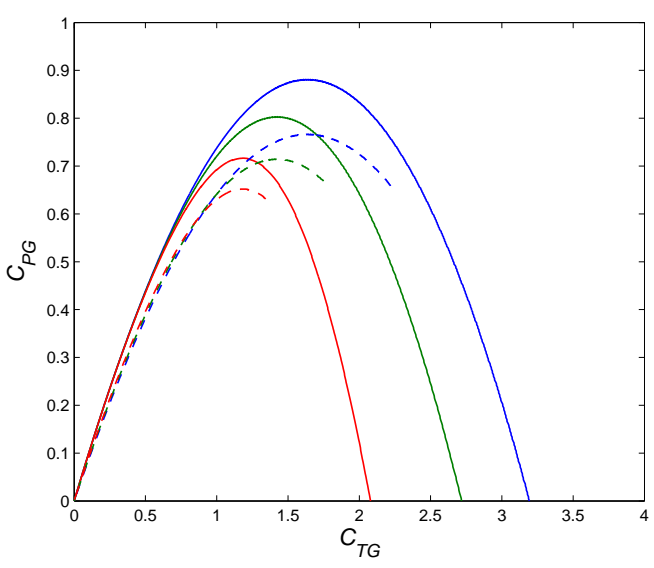

(c) Sixteen turbine array.

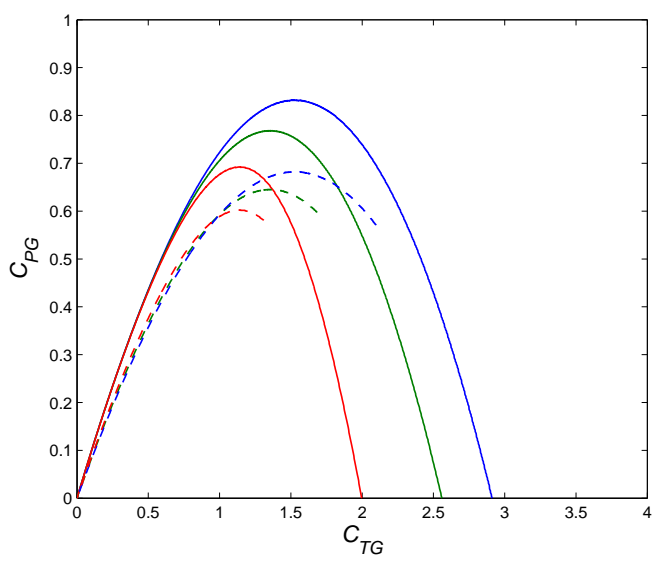

(b) Eight turbine array.

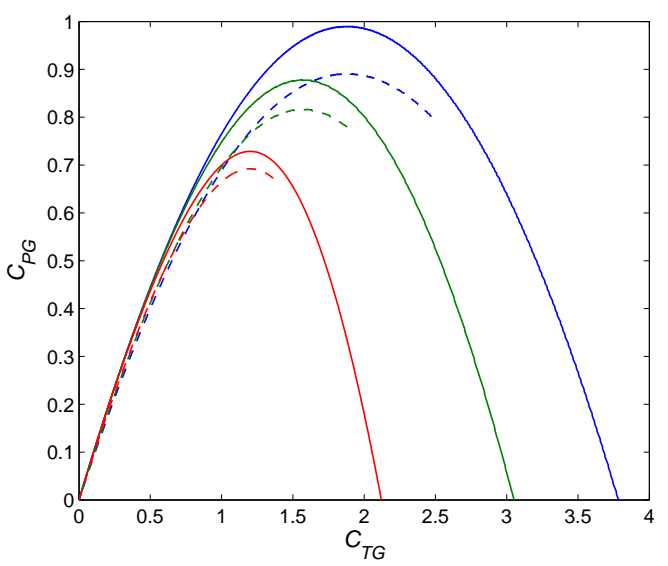

(d) Thirty-two turbine array.

Figure 10: Comparison between simulated (dashed lines) and analytically predicted (solid lines) power coefficient $C_{P G}$ vs. thrust coefficient $C_{T G}$ for arrays of different length and three local blockage ratios; $B_{L}=\frac{\pi}{32}$ (red), $B_{L}=\frac{\pi}{16}$ (green), and $B_{L}=\frac{\pi}{10}$ (blue).

simulations concerning the numerical stability and accuracy of the depth-averaged representation of the array, chiefly it is important to conserve both the blockage ratio and the momentum removed from the flow when using reduced-order models to ensure that the power available to the turbines is approximated as accurately as possible.

Analytic sub-model corrections for depth averaged simulations of energy extraction by an array of tidal turbines have been developed using rigid lid [5] and free surface [6] LMADT. The local efficiency, the ratio of the available power to the total power dissipated in the device-scale flow, may be used as a correction to the power removed from the depth-averaged simulation if the region in which the array is simulated is assumed to contain all device-scale flow events resulting from energy extraction. Local efficiency may be determined as a function of the device thrust coefficient, and therefore related to the array thrust through LMADT.

Power extracted from a flow by an array is significantly over predicted in a depth-averaged simulation when the device-scale flow is not modelled. For an eight device test array considered in this work, the depth-averaged simulation predicted a maximum power coefficient of $C_{P G, \max }=0.85$, compared to the 3D RANS simulation with $C_{P G, \max }=0.73$, a difference of $27 \%$. By comparison, applying the rigid lid analytic correction reduced the peak power coefficient from the simulation to $C_{P G, \max }=0.76$, an error of $4 \%$. The analytic correction may be applied during post-processing of the simulation, and thus it does not penalise the computational 
time of the depth-averaged simulation.

Local efficiency variation with thrust coefficient is dependent on the local blockage ratio. Local efficiency is negatively correlated with thrust coefficient, and declines more steeply for low blockage ratios than higher blockage ratios. This is because the thrust that can be applied to the flow by devices in low blockage arrays is limited by the tendency of the flow to bypass the devices as the resistance of the devices increases. The analytic correction thus plays a much more important role in estimating the power available to low blockage arrays rather than high blockage arrays, where the errors are typically less than $10 \%$.

Agreement between corrected depth-averaged simulations and analytic models for partial arrays is affected by the number of turbines in the array and the local blockage ratio. It is conceptually useful to divide the array into two sections, consisting of the inboard devices, in which the resistance to flow bypass is approximately equal either side of the devices, and the outboard devices, for which the resistance to bypass flow is substantially skewed due to the array end effects and cross-stream flows.

The flow around inboard devices is well approximated by the assumption of a uniform upstream flow, as assumed in analytic models. The uniform flow assumption however does not provide good agreement with the flow around outboard devices. As the number of turbines in the array increases, the ratio of inboard to outboard devices increases, and consequently agreement with analytic models improves. The influence of the skewed flow is greater for higher local blockage ratios where increased flow confinement allows a larger pressure drop to be established across the turbines, and hence a greater thrust applied to the flow at peak power. Flow diversion around outboard devices increases at higher thrust coefficients, worsening agreement with the analytic theory. Conversely, to achieve peak power for turbines in a low local blockage array a lower level of thrust is applied to the flow due to the relative ease with which flow bypasses the devices, reducing the outboard turbine array end effect and improving agreement with analytic theory, albeit at a lower peak power coefficient than for a higher local blockage array.

\section{Acknowledgements}

The authors wish to acknowledge the support of the Oxford Martin School. The authors are grateful to T. Nishino for providing three-dimensional data for comparison in this work.

[1] Adcock, T. A. A., Draper, S., Houlsby, G. T., Borthwick, A. G. L. and Serhadglığlu, S. [2014], 'Tidal stream power in the Pentland Firth - long term variability, multiple constituents, and capacity factor', Proceedings of the Institute of Mechanical Engineers Part A: Journal of Power and Energy 228(8), 854861.

[2] Adcock, T. A. A., Draper, S. and Nishino, T. [2015], 'Tidal power generation - A review of hydrodynamic modelling', Proceedings of the Institute of Mechanical Engineers Part A: Journal of Power and Energy pp. $1-17$.

[3] Draper, S., Houlsby, G. T., Oldfield, M. L. G. and Borthwick, A. G. L. [2010], 'Modelling tidal energy extraction in a depth-averaged coastal domain', IET Renewable Power Generation 4, 545-554.

[4] Elghali, S. E. B., Balme, R., Saux, K. L., Benbouzid, M. E.-H., Charpentier, J. F. and Hauville, F. [2007], 'A simulation model for the evaluation of the electrical power potential harness by a marine current turbine', IEEE Journal of Ocean Engineering 32(4), 786-797.

[5] Garrett, C. and Cummins, P. [2007], 'The efficiency of a turbine in a tidal channel', Journal of Fluid Mechanics 588, 243-251.

[6] Houlsby, G. T. and Vogel, C. R. [2016], 'The power available to tidal turbines in an open channel flow', ICE Energy (Ahead of print).

[7] Kramer, S. C. and Piggott, M. D. [2016], 'A correction to the enhanced bottom drag parameterisation of tidal turbines', Renewable Energy 92, 385-396. 
[8] Nishino, T. and Willden, R. H. J. [2012a], 'Effects of 3-D channel blockage and turbulent wake mixing on the limit of power extraction by tidal turbines', International Journal of Heat and Fluid Flow 37, 123135.

[9] Nishino, T. and Willden, R. H. J. [2012b], 'The efficiency of an array of tidal turbines partially blocking a wide channel', Journal of Fluid Mechanics 708, 596-606.

[10] Nishino, T. and Willden, R. H. J. [2013], 'Two-scale dynamics of flow past a partial cross-stream array of tidal turbines', Journal of Fluid Mechanics 730, 220-244.

[11] Plew, D. R. and Stevens, C. L. [2013], 'Numerical modelling of the effect of tidal turbines on currents in tidal channels - Tory Channel, New Zealand', Renewable Energy 57, 269-282.

[12] Vogel, C. R., Willden, R. H. J. and Houlsby, G. T. [2016], 'Effect of free surface deformation on the extractable power of a finite width turbine array', Renewable Energy 88, 317-324.

[13] Whelan, J. I., Graham, J. M. R. and Pieró, J. [2009], 'A free surface and blockage correction for tidal turbines', Journal of Fluid Mechanics 624, 281-291. 